\title{
Efectos de la melatonina sobre la macro-arquitectura del sueño en pacientes con demencia tipo Alzheimer
}

\author{
Manuel Alejandro Cruz-Aguilar, ${ }^{1}$ Ignacio Ramírez-Salado, ${ }^{2}$ Carlos Cruz-Ulloa, ${ }^{3}$ Gloria Benítez-King ${ }^{4}$
}

Artículo original

\section{SUMMARY}

The objective of the present study was to evaluate the $5 \mathrm{mg}$. melatonin effects on the sleep macro-architecture in eight patients with middle to moderate Alzheimer's disease (DTA). Using the polysomnographic technique (PSG), we made a simple-blind, non-randomized, controlled with placebo study. The PSG was carried out according to the following order: night 1: placebo administration; night 2 and 3: continues melatonin administration. In the first night with melatonin treatment, the sleep latency to the first episode of Stage 2, Delta and REM sleep, was significantly diminished as compared with placebo ( $\leq .05)$. No significant difference in total time of each sleep stage and sleep efficiency was observed. Nevertheless, a tendency to diminish the total time of nocturnal wake and increase of the total sleep time in the second night with melatonin treatment was observed. We conclude that melatonin can improve sleep in patients with middle to moderate DTA.

Key words: Alzheimer, sleep, melatonin.

\section{RESUMEN}

El objetivo del presente estudio fue determinar los efectos de $5 \mathrm{mg}$. de melatonina de liberación inmediata sobre la macro-arquitectura del sueño en ocho pacientes con diagnóstico de Demencia Tipo Alzheimer (DTA) de media a moderada. Utilizando la técnica polisomnográfica (PSG) se realizó un estudio simple ciego, no aleatorio, controlado con placebo. Los registros PSG se llevaron a cabo de la siguiente manera: Noche 1: administración de placebo; noche 2 y 3 : administración continua de melatonina $(5 \mathrm{mg})$. Observamos que el tratamiento con melatonina durante la primera noche de administración disminuyó significativamente la latencia de la fase 2, del sueño de ondas delta y el sueño de MOR al ser comparadas con el placebo $(\mathrm{P} \leq .05)$. No se observaron diferencias significativas en el tiempo total de cada fase de sueño; tampoco se observaron diferencias en la eficiencia del sueño en presencia de la melatonina. Sin embargo se observó una tendencia a la disminución del tiempo total de vigilia y un aumento del tiempo total de sueño, principalmente durante la segunda noche de tratamiento. Concluimos que la melatonina puede mejorar el sueño en pacientes con DTA de media a moderada.

Palabras clave: Alzheimer, sueño, melatonina.

\section{INTRODUCCIÓN}

La demencia tipo Alzheimer (DTA) es el principal tipo de demencia que se presenta en personas de la tercera edad. En México afecta al $35 \%$ de la población de 65 años de edad y es la cuarta causa principal de muerte en el país, después de las enfermedades del corazón, del cáncer y de la diabetes. ${ }^{1}$

Este tipo de demencia inicia aproximadamente a partir de los 55 años de edad, con una pérdida progresiva de la memoria, cambios en la personalidad y comportamiento desinhibido. Asimismo se alteran funciones cognitivas como el lenguaje, el pensamiento abstracto, así como el re- conocimiento de personas y lugares. ${ }^{2}$ En cuanto a las funciones fisiológicas básicas, se ha observado que el sueño se ve afectado en este tipo de demencia. En pacientes con DTA se ha observado disminución o ausencia del sueño Delta, incremento en la vigilia nocturna, aumento en la somnolencia diurna y disminución en el tiempo total del sueño nocturno. En estadios tardíos de la enfermedad se han observado alteraciones en el ritmo circadiano del sueño, latencia al sueño MOR alargada y un descenso del porcentaje de esta fase de sueño. ${ }^{3-6}$ Existe suficiente evidencia de que las alteraciones del sueño se correlacionan con un mayor compromiso de la memoria, al igual que con el descenso de la cognición. Es

\footnotetext{
Laboratorio de Sueño. Dirección de Investigación en Neurociencias. Instituto Nacional de Psiquiatría Ramón de la Fuente Muñiz.

Departamento de Cronobiología. Dirección de Investigaciones en Neurociencias. Instituto Nacional de Psiquiatría Ramón de la Fuente Muñiz.

3 Departamento de Neurología. Clínica "Samuel Ramírez Moreno".

4 Departamento de Neurofarmacología. Subdirección de Investigaciones Clínicas. Instituto Nacional de Psiquiatría Ramón de la Fuente Muñiz.

Correspondencia: Manuel Alejandro Cruz-Aguilar. Laboratorio de Sueño. Dirección de Investigaciones en Neurociencias. Instituto Nacional de Psiquiatría Ramón de la Fuente Muñiz. Calz. México-Xochimilco 101, San Lorenzo Huipulco, Tlalpan, 14370, México, DF. Tel: (01-55) 4160-5112/5113. E-mail: macrag@gmail.com
}

Recibido: 24 de septiembre de 2012. Aceptado: 13 de febrero de 2013. 
por ello que la optimización del manejo de los trastornos de sueño en estos pacientes es una prioridad.

El daño de las estructuras y vías neuronales relacionadas con los mecanismos de instalación del sueño, es la causa más directa de los cambios en el ciclo sueño-vigilia observados en la DTA. El reloj biológico que ejerce el control de los ritmos circadianos; incluyendo el del sueño, es el núcleo supra-quiasmático (NSQ). ${ }^{7,8}$ Se ha sugerido que este núcleo se encuentra importantemente afectado en la DTA. ${ }^{4}$ El NSQ transmite la información luminosa captada por la retina hacia la glándula pineal donde es secretada la melatonina (5-metoxy-N-acetiltriptamina). ${ }^{8}$ Esta indolamina es la hormona más relacionada con la regulación de los ritmos circadianos y se ha demostrado que tiene efectos sobre la instalación de los mecanismos del sueño. ${ }^{9}$ Existe evidencia de que la melatonina en sujetos sin demencia reduce la latencia al sueño, promueve una distribución temporal menos fragmentada, incrementa el tiempo total del sueño, su eficiencia y mejora su calidad. ${ }^{10-15}$

En años recientes se ha incrementado el uso de la melatonina en el tratamiento de alteraciones del ciclo sueño-vigilia. En humanos, se ha observado que dosis de 3 a $5 \mathrm{mg}$. mantienen la sincronización del ritmo circadiano en un ciclo de 24 horas, mejorando a su vez el sueño. ${ }^{9}$ Sin embargo la información existente acerca de los efectos de la melatonina sobre el sueño en pacientes con diagnóstico de DTA es limitada. Se ha propuesto que esta hormona en personas con DTA alarga los periodos de sueño y mejora el ciclo sueño-vigilia. ${ }^{16,17} \mathrm{Sin}$ embargo, no se conocen con exactitud los efectos de la melatonina sobre la macro-arquitectura del sueño en la DTA, ya que la mayor parte de los estudios realizados hasta ahora, han utilizado técnicas como la actimetría, la cual genera información sobre la cantidad de movimientos del paciente en el periodo de $24 \mathrm{hrs}$, y los cuestionarios de sueño que permiten conocer la apreciación subjetiva del paciente con respecto a la calidad y hábitos de su sueño. Sin embargo, la técnica polisomnográfica (PSG) es actualmente el estándar científico más preciso que existe para evaluar de manera óptima la distribución temporal de los distintos estadios de sueño y vigilia. Asimismo, esta técnica permite explorar la acción de los fármacos sobre la actividad cortical, ya que compromete el registro de la actividad electroencefalográfica (EEG). Con base en lo anterior, el objetivo del presente estudio fue determinar los efectos de la administración oral de $5 \mathrm{mg}$ de melatonina sobre la organización temporal de las fases de sueño nocturno (macro-arquitectura), en personas con diagnóstico de DTA de media-moderada, utilizando la técnica PSG.

\section{MATERIALES Y MÉTODOS}

\section{Sujetos}

Se estudiaron ocho pacientes que acudían al Departamento de Neurología del Hospital "Samuel Ramírez Moreno”, en la Ciudad de México. Los pacientes tenían una edad promedio de 65 años.

El diagnóstico de DTA se realizó a través de una exploración neurológica, la cual permitió establecer el diagnóstico de síndrome demencial. Posteriormente se realizaron pruebas para-clínicas, con el objetivo de realizar el diagnóstico de demencias reversibles. Para este procedimiento se aplicaron las siguientes mediciones: biometría hemática, química sanguínea, pruebas de funcionamiento tiroideo y hepático, TAC simple contrastada, evaluación de los factores isquémicos en demencia a través de la escala de Hachinski y se realizó además un estudio de electroencefalografía.

Una vez excluidas las demencias reversibles, se aplicó una escala diseñada en nuestro laboratorio para evaluar las funciones mentales superiores, en la cual debía observarse como mínimo, un deterioro en el lenguaje, praxis y gnosis utilizando los criterios de enfermedad de probable o posible DTA de la Asociación de la Enfermedad de Alzheimer y desórdenes relacionados (NINCDS-ADRDA).${ }^{18}$ La severidad de la demencia fue determinada mediante la prueba Mini-mental, ${ }^{19}$ y se consideró como criterio de inclusión que todos los sujetos obtuvieran un puntaje promedio correspondiente de severidad de media a moderada (10-22 puntos). Así mismo se consideró como criterio de inclusión que los sujetos presentaran alteraciones del sueño, lo cual se evaluó a través de una entrevista clínica diseñada en nuestro laboratorio. Esta entrevista se aplicó a las personas que pasan la noche habitualmente con los pacientes (cuidadores). Ninguno de los pacientes recibió previamente tratamiento farmacológico a base de inhibidores de acetilcolina o algún otro medicamento con acción psicotrópica.

Se obtuvo el consentimiento informado de los cuidadores de los pacientes vía consulta externa. De acuerdo con los lineamientos establecidos en la Declaración de Helsinki y los lineamientos del National Institutes of Health de Estados Unidos de Norte América, el estudio fue aprobado por la comisión de ética en investigación humana del Instituto Nacional de Psiquiatra Ramón de la Fuente Muñiz.

\section{Diseño del estudio}

Se realizó un estudio simple ciego, no aleatorio, controlado con placebo. A cada paciente se le realizaron cuatro registros PSG nocturnos, utilizando la primera noche como periodo de habituación a las condiciones de registro, una noche en la cual se administró placebo $(\mathrm{P})$ y durante dos noches continuas (M1-M2) se administraron 5mg. de melatonina vía oral, en una sola dosis. Se utilizó melatonina de liberación inmediata. La melatonina y el placebo fueron administrados una hora antes de que los pacientes se dispusieran a dormir (aproximadamente a las 9:00pm). El orden de los tratamientos ( $\mathrm{P}$ y M1-M2) fue contrabalanceado. Los registros PSG del tratamiento M1-M2 se realizaron con una semana de intervalo de la sesión de placebo. Para fines esta- 
dísticos todos los pacientes fueron su propio control, y todos ellos formaron parte de los distintos grupos del diseño experimental (P, M1-M2).

\section{Métodos de evaluación}

Registros PSG. Se colocaron electrodos en las derivaciones bipolares O1-O2, C4-A2, C3-A1, T3-F7, T4-F8, F3-F4 del sistema internacional 10-20 para EEG humana. Se colocaron de manera superficial electrodos en ambos cantos oculares para obtener el electrooculograma (EOG) y se colocaron de la misma manera electrodos en el músculo mentalis para adquirir el electromiograma (EMG). Se registraron nueve horas continuas de registro PSG que se iniciaron a las 22:00pm y que culminaron a las 7:00am del día siguiente. Los registros fueron realizados en una cámara diseñada especialmente para PSG en humanos, sono-amortiguada, eléctricamente aislada y con circuito cerrado de audio y video. Se utilizó un sistema digital para PSG (Grass, Co USA, modelo 15), con un rango de muestreo de $512 \mathrm{~Hz}$, utilizando un filtro para bandas de 11 a $35 \mathrm{~Hz}$.

La distribución temporal de los estadios conductuales del sueño y la vigilia, se evaluó en épocas electroencefalográficas de 30 segundos, a partir de los criterios internacionales establecidos por Rechtschaffen y Kales para PSG. ${ }^{20} \mathrm{La}$ macro-arquitectura del sueño fue analizada a partir de latencias del sueño; que fue entendida como el tiempo (min) que tarda en aparecer el primer episodio de cada una de las fase de sueño. Asimismo se analizó el tiempo total de cada una de las fases de sueño y su eficiencia; la cual fue el porcentaje de sueño del tiempo total de registro.

\section{Análisis estadístico}

Las variables antes descritas fueron estadísticamente comparadas utilizando la prueba $t$ de Student. Se realizaron comparaciones entre las condiciones P y M1, y P y M2. Para realizar el análisis se utilizó el software SigmaPlot 11.0. Las diferencias se consideraron estadísticamente significativas con una $p \leq .05$.

\section{RESULTADOS}

\section{Observaciones cualitativas}

Al evaluar visualmente la distribución temporal de los distintos estadios de sueño y vigilia, observamos que dos de nuestros pacientes no presentaron sueño delta durante la noche de habituación y placebo. Sin embargo, esto se ve contrarrestado en presencia de la melatonina, ya que todos nuestros pacientes presentaron varios episodios de sueño delta distribuidos en las ocho horas de estudio, durante ambas noches en que se administró la melatonina. Durante la noche de placebo, en todos nuestros pacientes observamos una gran cantidad de movimientos corporales de corta duración que fragmentan principalmente la continuidad del sueño no-MOR. Sin embargo estos movimientos disminuyeron progresivamente a través de las noches de tratamiento con melatonina (figura 1). También observamos bruxismo durante el sueño no-MOR en tres de nuestros pacientes en todas las noches de registro.

\section{Análisis cuantitativo}

El cuadro 1 muestra los resultados del análisis estadístico. Las figuras 2, 3 y 4 representan gráficamente estos valores, los cuales son expresados como la media \pm la desviación estándar (DS). Los resultados de las pruebas $t$ muestran que entre las latencias de las condiciones P y M1 existen diferencias estadísticamente significativas, observándose una disminución del tiempo de instalación de los primeros episodios de la fase 2 del sueño no-MOR ( $p=0.044)$, del sueño delta $(p=0.017)$ y del sueño MOR $(p=0.032)$ (figura 2$)$. Sin embargo no se observaron diferencias significativas de las latencias del sueño entre las condiciones P y M2. En cuanto a la eficiencia del sueño, no se observaron diferencias signi-
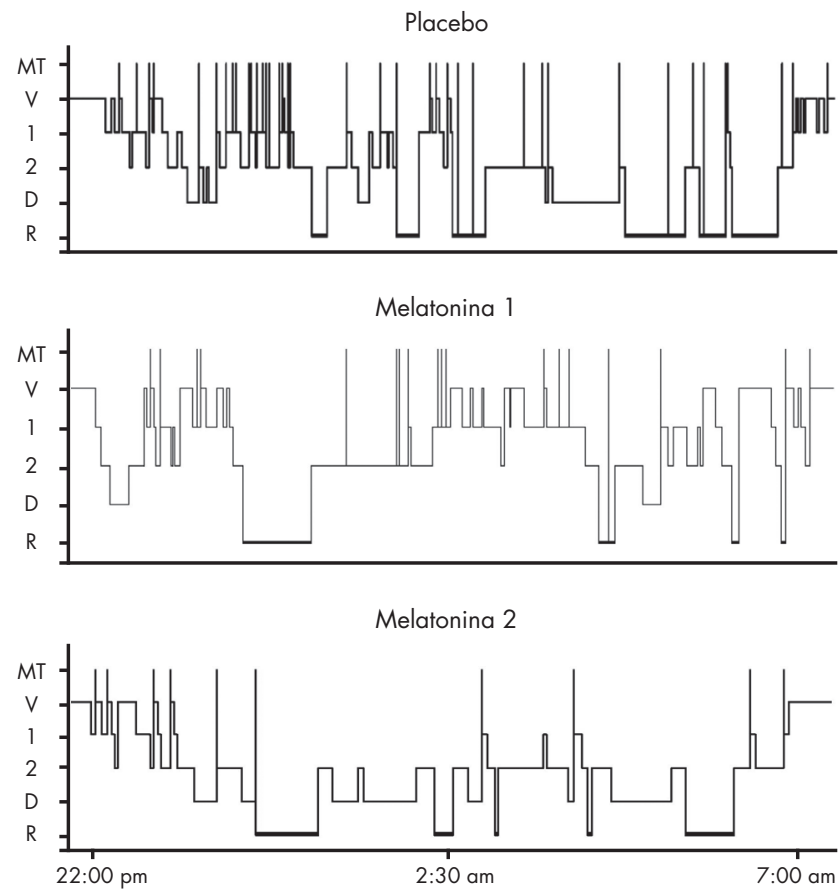

Figura 1. Hipnogramas representativos de la macro-arquitectura del sueño observada en la condición de placebo $(P)$ y en la primera (M1) y segunda (M2) noche de administración de la melatonina. La imagen muestra el caso de uno solo de nuestros pacientes. En el eje $Y$ se indica cada uno de los estadios evaluados, el eje $X$ representa las nueve horas de registro PSG. Las líneas horizontales de la gráfica representan el tiempo de cada estadio de sueño y vigilia. Las líneas verticales indican el cambio de fase. MT: movimiento, 1: fase 1 del sueño no-MOR, 2: fase 2 del sueño no-MOR, D: sueño DELTA, R: sueño MOR. 
Cuadro 1. Valores numéricos de la macro-arquitectura de sueño. Diferencias entre las condiciones placebo (P) y melatonina 1 (M1), y placebo y melatonina 2 (M2). Los valores son expresados como la media \pm desviación estándar (DS). Se muestran las probabilidades ( $p$ ) y los valores † de las comparaciones por grupo utilizando la Prueba t de student $(n=8)$. Los parámetros están expresados en minutos, excepto la eficiencia, la cual está representada como porcentaje. Las diferencias se consideraron estadísticamente significativas con una $\mathrm{p} \leq .05$.

\begin{tabular}{|c|c|c|c|c|c|c|c|c|}
\hline & \multicolumn{2}{|c|}{ Placebo } & \multicolumn{2}{|c|}{ Melatonina 1} & \multicolumn{2}{|c|}{ Melatonina 2} & \multirow[b]{2}{*}{$t$} & \multirow[b]{2}{*}{$P$} \\
\hline & Media & DS & Media & DS & Media & DS & & \\
\hline Eficiencia \% & 77.22 & 15.76 & 83.06 & 11.44 & 85.36 & 10.21 & & \\
\hline $\begin{array}{l}\text { Latencias (min) } \\
\text { - Fase } 1 \\
\text { - Fase } 2 \\
\text { - Sueño Delta } \\
\text { - Sueño Mor }\end{array}$ & $\begin{array}{r}17.93 \\
34.75 \\
210.33 \\
172.40\end{array}$ & $\begin{array}{r}10.24 \\
24.15 \\
169.12 \\
109.80\end{array}$ & $\begin{array}{l}15.43 \\
15.25^{*} \\
33.71^{*} \\
76.50^{*}\end{array}$ & $\begin{array}{r}9.31 \\
5.95 \\
15.69 \\
30.50\end{array}$ & $\begin{array}{r}15.43 \\
30.43 \\
94.58 \\
128.93\end{array}$ & $\begin{array}{r}9.31 \\
20.08 \\
9.14 \\
85.82\end{array}$ & $\begin{array}{l}2.217 \\
2.988 \\
2.380\end{array}$ & $\begin{array}{l}.044 \\
.017 \\
.032\end{array}$ \\
\hline $\begin{array}{l}\text { Tiempo total (m } \\
\text { - Vigilia } \\
\text { - Fase I } \\
\text { - Fase } 2 \\
\text { - Sueño Delta } \\
\text { - Sueño Mor }\end{array}$ & $\begin{array}{r}89.93 \\
54.87 \\
221.87 \\
29.00 \\
56.66\end{array}$ & $\begin{array}{l}57.57 \\
37.34 \\
86.69 \\
36.41 \\
29.37\end{array}$ & $\begin{array}{r}72.75 \\
66.50 \\
248.06 \\
29.50 \\
76.50\end{array}$ & $\begin{array}{l}50.12 \\
35.78 \\
78.83 \\
18.79 \\
30.50\end{array}$ & $\begin{array}{r}57.75 \\
67.37 \\
212.87 \\
63.12 \\
85.00\end{array}$ & $\begin{array}{r}55.39 \\
75.71 \\
106.83 \\
51.86 \\
39.99\end{array}$ & & \\
\hline
\end{tabular}

* Diferencias significativas entre $\mathrm{P}$ y $\mathrm{Ml}$.

ficativas relacionadas con la melatonina en ninguna de las dos noches de tratamiento (figura 3).

En cuanto al tiempo total de cada fase de sueño las variaciones observadas no fueron estadísticamente significativas, sin embargo los resultados muestran una tendencia al incremento de la fase 1 en la condición M1 y M2, así mismo se observa un incremento del tiempo total de la fase $2 \mathrm{du}-$ rante la condición M1. El sueño delta incrementó sus valores durante la condición M2 principalmente. El sueño MOR mostró un aumento progresivo de sus valores a través de ambas condiciones de melatonina, así como el tiempo total

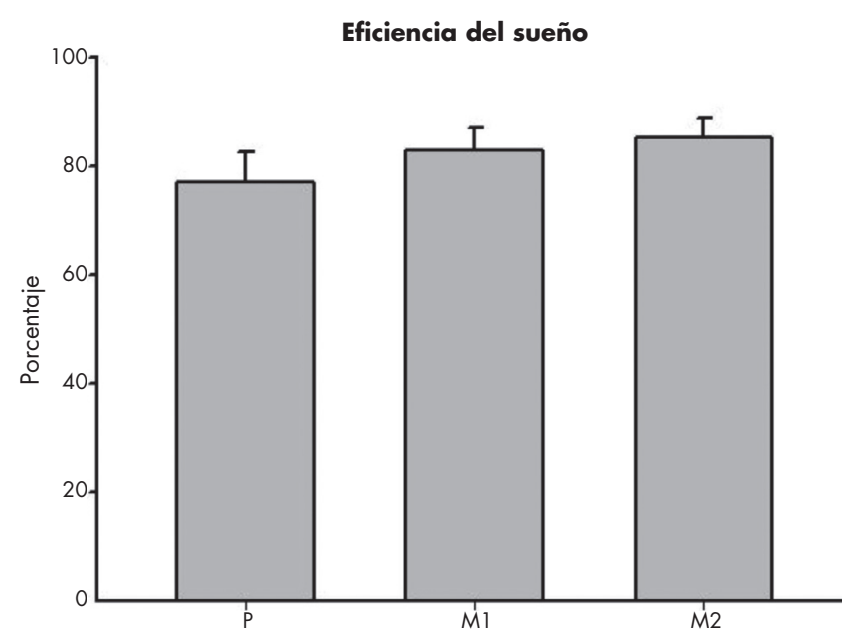

Figura 2. Latencias del primer episodio de las distintas fases de sueño. Eje $X=$ condición de placebo $(P)$, primera noche de administración de melatonina (M1) y segunda noche de administración de melatonina (M2). Eje $Y=$ tiempo ( $\mathrm{min}$ ) en que aparece el primer episodio de cada fase de sueño desde el inicio del registro PSG 19:00 pm aprox. *Diferencias significativas entre P y M1. de vigilia, el cual también disminuyó principalmente en la condición M2 (figura 4).

\section{DISCUSIÓN}

En este estudio se observó que la melatonina en pacientes con DTA disminuye significativamente la latencia de instalación de la fase 2 del sueño no-MOR, del sueño delta y del sueño MOR, principalmente durante la primera noche de administración. Así mismo se observó una tendencia a

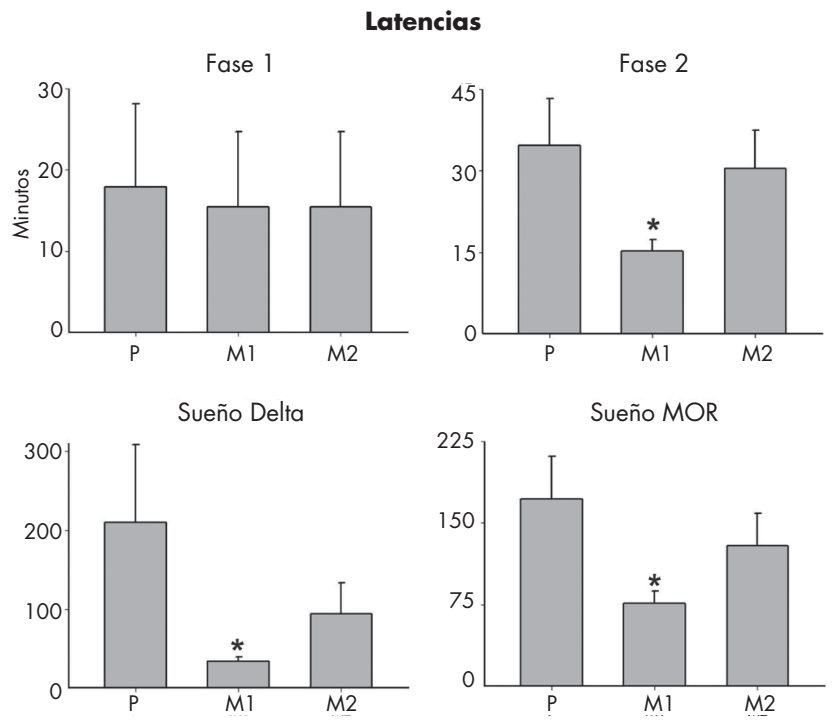

Figura 3. Eficiencia del sueño. Eje $X=$ condición de placebo $(P)$, primera noche de administración de melatonina (MI) y segunda noche de administración de melatonina (M2). Eje $Y=$ Porcentaje de sueño del tiempo total de registro. No se observaron diferencias estadísticamente significativas relacionadas con la melatonina. 

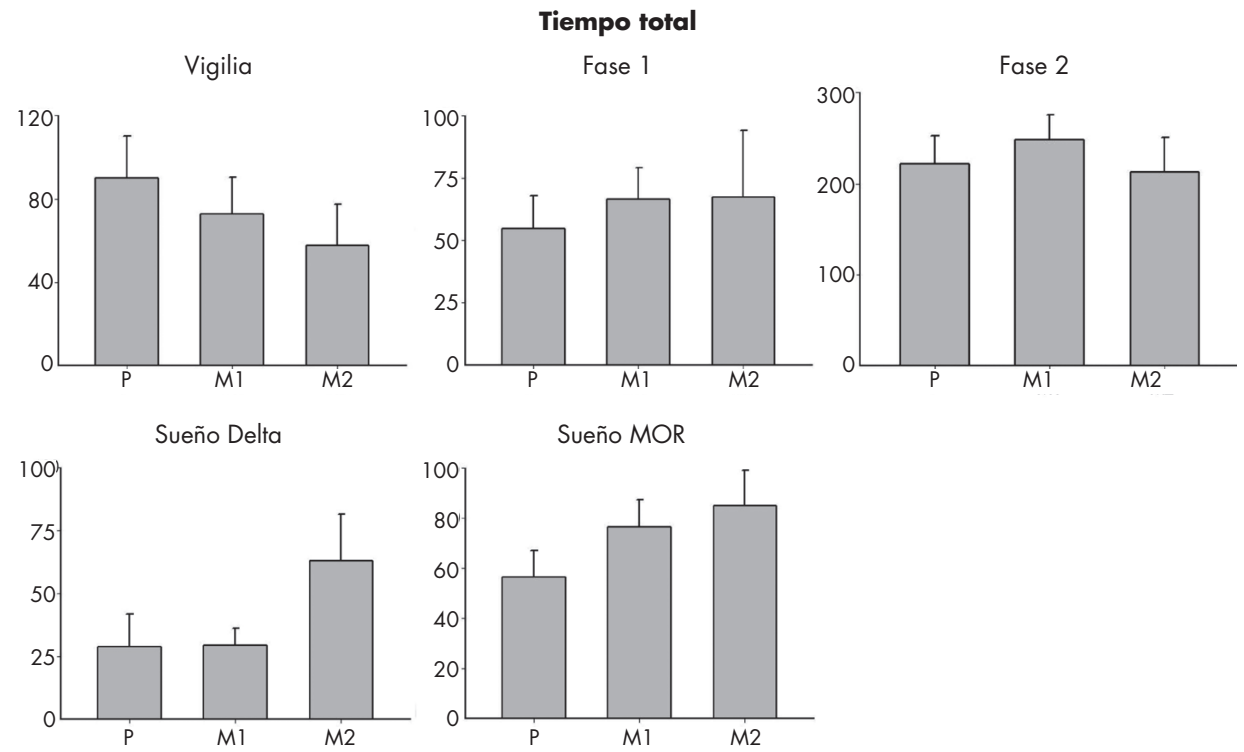

Figura 4. Tiempo total de las distintas fases de sueño y vigilia. Eje $X=$ condición de placebo (P), primera noche de administración de melatonina (M1) y segunda noche de administración de melatonina (M2). Eje $Y=$ tiempo total $(\mathrm{min})$ de cada fase de sueño y vigilia. No se observaron diferencias estadísticamente significativas relacionadas con la melatonina.

la disminución del tiempo total de vigilia y un aumento del tiempo total de sueño, principalmente la segunda noche de administración.

En cuanto a la disminución observada de las latencias, notamos que éstas se aproximan al tiempo normal reportado anteriormente en pacientes geriátricos sin demencia. ${ }^{21}$ Así mismo, el efecto de la pronta instalación del sueño provocado por melatonina en pacientes geriátricos sin demencia ha sido documentado anteriormente. Se ha observado que dosis de $3 \mathrm{mg}$ favorecen la pronta instalación del sueño no-MOR, ${ }^{17}$ tal como se observó en el presente estudio. Estos resultados sugieren que estructuras como el NSC, así como los circuitos tálamo-corticales y estructuras del tallo cerebral encargadas del control y regulación del sueño, aun en la DTA media a moderada, son capaces de reaccionar a la cascada de eventos que la melatonina ejerce para facilitar la instalación del sueño.

Actualmente no se conoce con precisión el mecanismo neurobiológico que explique la inducción de sueño producido por la melatonina, sin embargo existen distintos trabajos que apoyan la idea de que esta hormona induce el sueño a través de la modulación de los receptores a GABA. Se ha propuesto que dosis farmacológicas de melatonina ( $\leq 1 \mathrm{mg}$.) pueden interactuar directamente con los receptores a benzodiacepinas, los cuales ejercen sus efectos a través de la modulación GABAérgica. ${ }^{21-25}$ Esto sugiere que la interacción de la melatonina con el sistema GABAérgico podría estar relacionado con la activación de los mecanismos neurofisiológicos de instalación del sueño.

Asimismo, nuestros resultados muestran por primera vez que la melatonina ejerce efectos sobre la instalación del sueño MOR en personas con DTA. En este sentido, se sabe que el mecanismo que activa los diversos componentes del sueño MOR está formado por un grupo de neuronas de la protuberancia dorsal de naturaleza colinérgica. ${ }^{26-28} \mathrm{Sin}$ embargo, se ha descrito que un difuso grupo de neuronas del área pre-óptica ventro-lateral (VLPO) es capaz de inducir sueño MOR a través de sus proyecciones que inhiben la expresión de monoaminas del raphé dorsal (serotonininérgico) y el locus coeruleus (noradrenérgico). ${ }^{29-31}$ Las proyecciones inhibitorias hacia el VLPO por los componentes histaminérgicos, serotoninérgicos y noradrenérgicos del sistema de vigilia, utilizan el GABA que está presente en el $80 \%$ de las neuronas del VLPO. Si la melatonina modula la actividad GABAérgica, entonces es posible que las neuronas del VLPO sean activadas por esta hormona con una consecuente supresión de la vigilia, facilitando a su vez la instalación y la expresión del sueño MOR. A las neuronas del VLPO se les denomina neuronas activas durante el sueño (REM-on), las cuales inervan áreas que promueven la vigilia, como el núcleo tuberomamilar, el hipotálamo lateral, el raphé dorsal, el tegmento dorsolateral (LDT) y el tegmento pedúnculopontino (PPT). ${ }^{30,31}$ Es probable que el aumento de la actividad de las neuronas sueño activas, de naturaleza GABAérgica, inhiban áreas promotoras de la vigilia y así, induzcan el sueño MOR.

Es de interés señalar que la acción que ejerce la melatonina sobre el sueño de nuestros pacientes podría tener beneficios terapéuticos sobre el déficit de memoria y aprendizaje característicos de la DTA, sobre todo para el desempeño de estas funciones al día siguiente de haber administrado el fármaco, ya que se ha demostrado que el sueño MOR y la fase 2 
del sueño no-MOR se encuentran estrechamente relacionados con distintos procesos cognitivos que subyacen a la fijación y mantenimiento de la memoria y el aprendizaje. ${ }^{32-34}$ Asimismo, durante el sueño MOR tienen lugar procesos de restauración celular que no se observan en ningún otro momento del ciclo sueño-vigilia. Por otro lado, es de interés señalar que durante el sueño delta tienen lugar la secreción de hormona de crecimiento y la síntesis de neurotrofinas, ${ }^{21}$ procesos de restauración fisiológica que son sumamente importantes en la DTA. ${ }^{35}$

Se ha propuesto que el sueño está controlado por dos sistemas. Por una parte, el ritmo circadiano; el cual es la tendencia natural al sueño que varía a lo largo del día y se manifiesta principalmente en el sueño MOR. Por otro lado, se encuentra la homeostasis del sueño, la cual hace que el hombre sienta somnolencia por la noche. ${ }^{36}$ El sueño delta refleja fielmente este control homeostásico del sueño, ya que se ha demostrado que toda aquella situación que suponga un aumento de la vigilia previa al período de sueño supondrá un incremento en la proporción del sueño delta. ${ }^{24,36-39}$

Nuestros resultados sugieren que la melatonina ejerce acción sobre ambos procesos, ya que observamos una tendencia al aumento del tiempo total del sueño delta, así como una disminución de la latencia de esta fase de sueño y del sueño MOR. Estos resultados indican que la melatonina favorece que el organismo inicie los procesos de restauración y descanso a la hora más adecuada del ciclo luz-oscuridad natural, lo cual tiene gran valor terapéutico.

La notable tendencia al aumento del tiempo total de sueño y la disminución de la vigilia nocturna, sobre todo en la segunda noche de administración, indican que la melatonina puede promover mayor cantidad de sueño nocturno, lo cual tiene impacto directo en la calidad de vida de los pacientes y de las personas que viven y cuidan de ellos, ya que comúnmente estas personas se encuentran privadas de sueño a consecuencia de los distintos desórdenes del ciclo sueño-vigilia que presentan los pacientes con DTA.

Asimismo, nuestros resultados señalan que la melatonina posee una acción directa sobre los mecanismos de instalación del sueño en general, pero no sobre los de mantenimiento, ya que la eficiencia del sueño no muestra cambios significativos en presencia de la melatonina. Sin embargo, estos efectos limitados del fármaco podrían deberse al tipo de liberación utilizada. Dado que la melatonina tiene una vida media corta, la liberación prolongada tendría la posibilidad de activar los receptores cerebrales durante toda la noche mejorando la eficiencia del sueño, por lo que se sugiere utilizar en futuros estudios melatonina de liberación prolongada, con el objetivo de determinar si este tipo de presentación es conveniente para obtener mejores efectos terapéuticos en pacientes con DTA. Así mismo se sugiere realizar estudios PSG más completos, en los que se incluya registro de la actividad respiratoria y el electrocardiograma (ECG). Consideramos necesario realizar el análisis espectral de la actividad EEG en presencia de melatonina, ya que se ha demostrado que este tipo de análisis permite determinar los efectos homeostásicos precisos que ejerce esta hormona durante el sueño. ${ }^{38}$

\section{CONCLUSIONES}

En el presente estudio observamos que $5 \mathrm{mg}$ de melatonina facilitan la instalación del sueño en personas con DTA de media a moderada. Nuestros resultados sugieren que la melatonina podría ser utilizada como opción terapéutica para tratar problemas de sueño en personas que padecen este tipo de demencia.

\section{AGRADECIMIENTOS}

A la colaboración invaluable de Isidoro Camacho García. Los fármacos utilizados en el presente estudió fueron donados por el laboratorio Bio-quimed. El proyecto fue apoyado parcialmente con recursos del CONACyT, donativo No. 46593-M de la doctora Gloria Benítez King.

\section{REFERENCIAS}

1. Alanis-Niño G, Garza-Marroquin JV, Gonzáles-Arellano A. Prevalencia de demencia en pacientes geriátricos. Rev Med Inst Mex Seguro Soc 2008;46(1):27-32.

2. Bliwise DL. Sleep disorders in Alzheimer's disease and other dementias. Clin Cornerstone 2004;6(Supl 1A): S16-S28.

3. Montplaisir J, Petit D, Gauthier S, Gaudeau H et al. Sleep disturbances and EEG Slowing in Alzheimer's disease. Sleep Res Online 1998;1(4):147-151.

4. Vitello MV, Borson S. Sleep disturbances in patients with Alzheimer's disease: epidemiology, pathophysiology and treatment. CNS drugs 2001;15(10):777-796.

5. Hess CW. Sleep disorders and dementia. Schweiz Rundsch Med Prax 1997;86:1343-1349.

6. Onen F, Onen SH. Sleep rhythm disturbances in Alzheimer's disease. Rev Med Interne 2003;24:165-171.

7. Tobler I, Jaggi K, Borbély AA. Effects of melatonin and the melatonin receptor agonist S-20098 on the vigilance states, EEG spectra, and cortical temperature in the rat. J Pineal Res 1994;16:26-32.

8. Reiter RJ. Pineal melatonin: Cell biology of its synthesis and of its physiological interactions. Endoc Rev 1991;12:151-180.

9. Brzezinski A, Vangel MG, Wurtman RJ, Norrie G et al. Effects of exogenous melatonin on sleep: a meta-analysis. Sleep Med Rev 2005;9:41-50.

10. Anton-Tay F, Díaz JL, Fernandez-Guardiola A. On the effect of melatonin upon human brain. Its possible therapeutic implications. Life Sci 1971;10:841-850.

11. Brusco LI, Fainstein I, Marquez M, Cardinali DP. Effect of melatonin in selected populations of sleep-disturbed patients. Biol Signals Recept 1999;8:126-131.

12. Tzischinsky $O$, Degan $Y$, Laive $P$. The effects of melatonin on the timing of sleep in patients with delayed phase syndrome. En: Touitou Y, Ardent $\mathrm{J}$, Pevet $\mathrm{P}$ (eds). Melatonin and the pineal gland-from basic science and to clinical application. New York: Elsevier; 1993; pp.351-35.

13. Dijk DJ, Shanahan TL, Duffy JF. Melatonin, sleep consolidation, body temperature, slow wave and sleep spindle activity: Phase relations of the circadian rhythms during forced desyncrony. Sleep Res 1995;24(A): 162. 
14. Dijk DJ, Cajochen C. Melatonin and the circadian regulation of sleep initiation, consolidation, structure, and sleep EEG. J Biol Rhythm 1997;12:627-635.

15. Seabra MLV, Bignotto M, Pinto LR Jr, Tufik S. Randomized, doubleblind clinical trial, controlled with placebo, of the toxicology of chronic melatonin treatment. J Pineal Res 2000;29:193-200.

16. Asayama $K$, Yamadera $H$, Ito $T$, Suzuki $H$ et al. Double blind study of melatonin effects on the sleep-wake rhythm, cognitive and noncognitive functions in Alzheimer type dementia. J Nippon Med Sch 2003;70:334-341.

17. Cardinali DP, Brusco LI, Liberczuk C, Furio AM. The use of melatonin in Alzheimer's disease. Neuro Endocrinol Lett 2002;23(Supl 1):20-23.

18. Mckhann G, Drachman D, Folstein M, Katzman R et al. Clinical diagnosis of Alzheimer's disease: report of the NINCDS-ADRDA Work Group under the auspices of Departament of Health and human Services Task Force on Alzheimer's disease. Neurology 1984;34:939-944.

19. Folstein MF, Folstein SE, McHugh PR. Mini-mental state: a practical method for rating the cognitive state of patients for the clinical. J Psychiatr Res 11:189-198.

20. Rechtschaffen A, Kales AA. A manual of standardized terminology, techniques, and scoring system for sleep stages of human subjects. Bethesda, MD: US Department of Health, Education, and Welfare; 1968.

21. Reinoso Suárez F. The neurobiology of slow wave sleep. An R Acad Nac Med 1999;116:209-226.

22. Borbély AA, Achermann P. Sleep homeostasis and models of sleep regulation. En: Kyger $M$, Roth T, Dement $W$ (eds.). Principles and practice of sleep medicine. Philadelphia: Saunders; 2000: pp. 377-390.

23. Stankov B, Biella G, Panara C, Lucini V et al. Melatonin signal transduction and mechanism of action in the central nervous system: using the rabbit cortex as a model. Endocrinology 1992;130:2152-2159.

24. Borbély AA, Mattmann P, Loepfe M, Strauch I et al. Effect of benzodiazepine hypnotics on all-night sleep EEG spectra. Hum Neurobiology 1985;4:189-194.

25. Gaillard JM, Schulz P, Tissot R. Effects of three benzodiazepines (nitrazepam, unitrazepam and bromazepam) on sleep of normal subjects, studied with an automated sleep scoring system. Pharma-kopsychiatry 1973;6:207-217.

26. Maquet P, Peters J, Delfiore G, Aerts J et al. Regional cerebral haemodynamics during slow sleep and paradoxical sleep. Preliminary results of a positron emission tomography (PET) study. Sleep Res 1995;[A]24:89.

27. Maquet P, Péters JM, Aerts J, Delfiore G et al. Functional neuroanatomy of human rapid eye movement sleep and dreaming. Nature 1996; 383:163-166.

28. Calvo JM, Simón-Arceo K. Cholinergic enhancement of REM sleep from sites in the pons and amygdala. En: Lydic R, Baghdoyan HA (eds.). Handbook of behavioral state control: Cellular and molecular mechanisms. United States of America: CRC Press; 1999; pp. 391-406.

29. Lu J, Greco MA, Shiromani P, Saper CB. Effect of lesions of the ventrolateral preoptic nucleus on NREM and REM sleep. J Neurosci 2000;20:3830-3842.

30. Sherin JE, Elmquist JK, Torrealba F, Saper CB. Innervation of histaminergic tuberomammillary neurons by GABAergic and galaninergic neurons in the ventrolateral preoptic nucleus of the rat. J Neurosci 1998;18:4705-4721.

31. Szymusiak R, Alam N, Steininger TL, McGinty D. Sleep-waking discharge patterns of ventrolateral preoptic/anterior hypothalamic neurons in rats. Brain Res 1998;803:178-188.

32. Mignot E. Why we sleep: The temporal organization of recovery. PLoS Biol 2004;6(4):0661-0669.

33. Horne JA, McGrath MJ. The consolidation hypothesis for REM sleep function: Stress and other confounding factors. A review. Biol Psychol 1984;18:165-184.

34. Rotenberg VS. Sleep and memory: The influence of different sleep stages on memory. Neurosci Bio Behav Rev 1992;16:497-502.

35. Domínguez-Alonso A, Ramírez-Rodríguez G, Benitez-King G. Melatonin increases dendritogenesis in the hilus of hippocampal organotypic cultures. J pineal Res 2012;52:427-436.

36. Borbély AA. A two process model of sleep regulation. Hum Neurobiol 1982;1:195-204.

37. Dijk DJ, Brunner DP, Borbély AA. Time course of EEG power density during long sleep in humans. Am J Physiol 1990;258:650-661.

38. Achermann P, Dijk DJ, Brunner DP, Borbély AA. A model of human sleep homeostasis based on EEG slow wave activity: quantitative comparison of data and simulations. Brain Res Bull 1993;3:97-113.

39. Muñoz AI, Pérez-Martínez DA, Villalibre-Valderrey I. El papel del sueño de ondas lentas en la regulación homeostática del sueño. Rev Neurol 2002;34:211-215.

Artículo sin conflicto de intereses 